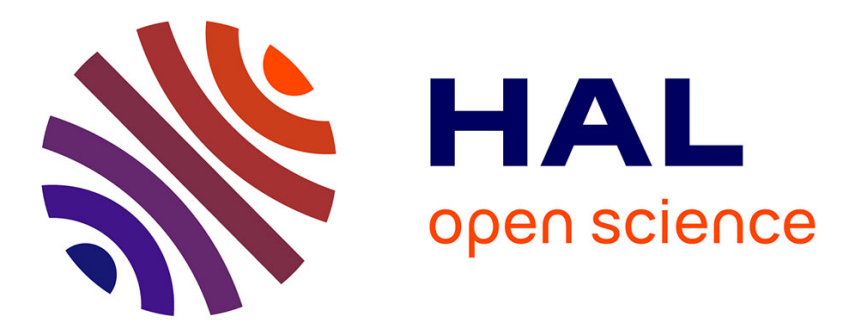

\title{
Quantum eigenlogic observables applied to the study of fuzzy behaviour of Braitenberg vehicle quantum robots
} Zeno Toffano, François Dubois

\section{To cite this version:}

Zeno Toffano, François Dubois. Quantum eigenlogic observables applied to the study of fuzzy behaviour of Braitenberg vehicle quantum robots. Kybernetes, 2019, 48 (10), pp.2307-2324. 10.1108/K11-2018-0603 . hal-02095400

\section{HAL Id: hal-02095400 \\ https://hal-centralesupelec.archives-ouvertes.fr/hal-02095400}

Submitted on 31 Mar 2020

HAL is a multi-disciplinary open access archive for the deposit and dissemination of scientific research documents, whether they are published or not. The documents may come from teaching and research institutions in France or abroad, or from public or private research centers.
L'archive ouverte pluridisciplinaire HAL, est destinée au dépôt et à la diffusion de documents scientifiques de niveau recherche, publiés ou non, émanant des établissements d'enseignement et de recherche français ou étrangers, des laboratoires publics ou privés. 


\title{
Quantum Eigenlogic Observables applied to the study of Fuzzy Behaviour of Braitenberg Vehicle Quantum Robots
}

\author{
Zeno Toffano \\ CentraleSupélec - Laboratoire des signaux et systèmes CNRS - Université Paris- \\ Saclay, Gif-sur-Yvette, France \\ François Dubois \\ CNAM - Association Francaise de Science des Systemes, Paris, France
}

\begin{abstract}
Purpose -The quantum-like logical formulation Eigenlogic, using logical quantum observables is applied to behavioural analysis. Agents, represented by Braitenberg vehicles, are investigated in the context of the quantum robot paradigm. The agents are processed through quantum logical gates with fuzzy and multivalued inputs, this permits to enlarge the behavioural possibilities and the associated decisions for these simple vehicles.
\end{abstract}

Design/methodology/approach - In Eigenlogic the eigenvalues of the observables are the truth-values and the associated eigenvectors are the logical interpretations of the propositional system. Logical observables belong to families of commuting observables for binary logic and many-valued logic. By extension, a fuzzy logic interpretation is proposed by using vectors outside the eigensystem of the logical connective observables. The fuzzy membership function is calculated by the quantum mean value (Born rule) of the logical projection operators and is associated to a quantum probability. The methodology of this paper is based on quantum measurement theory.

Findings - Fuzziness arises naturally when considering systems described by state vectors not in the considered logical eigensystem. These states correspond to incompatible and complementary systems outside the realm of classical logic. Considering these states allows the detection of new Braitenberg Vehicle behaviors related to identified emotions these are linked to quantum-like effects.

Research limitations/implications - The method does not deal at this stage with first-order logic and is limited to different families of commuting logical observables. An extension to families of logical non-commuting operators associated to predicate quantifiers could profit of the "quantum advantage" due to effects such as superposition, parallelism, non-commutativity and entanglement. This direction of research has a variety of applications, including robotics.

Practical implications - The purpose of this research is to show the multiplicity of behaviors obtained by using fuzzy logic along with quantum logical gates in the control of simple Braitenberg Vehicle agents. By changing and combining different quantum control gates one can tune small changes in the vehicle's behaviour, and hence get specific features around the main basic robot's emotions.

Originality/value - New mathematical formulation for propositional logic based on linear algebra. This methodology demonstrates the potentiality of this formalism for behavioural agent models (quantum robots).

\section{Keywords:}

Multivalued logic, fuzzy logic, quantum gates, quantum modelling, quantum robots, Braitenberg vehicles. 


\section{Introduction}

George Boole proposed a mathematical symbolic formulation using the two numbers $\{0,1\}$ representing respectively the "false" or "true" character of a proposition (Boole 1847). An idempotent symbol $x$, which verifies the equation: $x^{2}=x$, can take only two possible values: 0 and 1 , this equation was considered by Boole the "fundamental law of thought". The associated formulation for logic is operational as pointed out by Theodore Halperin (Halperin 1981) where the symbol $x$ acts as a selection operator on classes. As will be emphasized here the algebra of idempotent symbols can also be interpreted as a set of commuting projection operators in linear algebra.

Propositional logic is a basic tool for interacting with facts (Tatsachen) as summarized by the sixteen truth functions (reproduced hereafter in Table 1) by Ludwig Wittgenstein in the Tractatus at prop. 5.101 (Wittgenstein 1921) and concluded at prop. 5.6: "The limits of my language mean the limits of my world." and at prop. 5.61: "Logic pervades the world: the limits of the world are also its limits." If language sets the limits to the world, then the language available limits robotics. What is exposed hereafter is that quantum theory and is associated methods can seamlessly reproduce and extend propositional logic. Some definitions of propositional logic are reminded at Appendix A.

John von Neumann was the first to associate quantum projection operators with logical propositions, in (von Neumann 1932) (Ch. III: The Quantum Statistics, Sect 5: Projections as Propositions, p.247), which subsequently was formalized in an independent discipline: Quantum Logic (Birkhoff \& Von Neumann 1936). He also introduced the formalism of the density operator in quantum mechanics where a pure quantum state $|\psi\rangle$ can be represented by its associated projection operator: $\boldsymbol{\rho}=|\psi\rangle\langle\psi|$, which is a ray (a rank-1 idempotent projection operator spanning a one-dimensional subspace). All these concepts lay at the foundations of quantum theory.

Quantum theory breaks with classical physics concepts but at the same time builds on major findings in classical physics. It is considered the most successful theory of physics so far and is currently expanding its realm in other fields of science, including natural, technological (Nielsen \& Chuang 2000) and social sciences (Haven \& Khrennikov 2013).

In this paper a linear algebraic method is used, for binary, multi-valued and fuzzy logic using quantum observables in Hilbert space. All logical connectives have their corresponding observable where the truth values correspond to the eigenvalues. In this way propositional logic can be formalized by using tensor combinations of elementary quantum observables. The outcome of a "measurement" of a logical observable will give the truth value of the associated logical proposition, and becomes interpretable when applied to vectors of its eigenspace, leading to an original insight into the quantum measurement postulate.

In this approach fuzzy logic arises naturally when considering vectors outside the eigensystem. The fuzzy membership function is obtained by the quantum mean value (Born rule) of the corresponding logical projection observable on these vectors. Fuzziness arises because of the quantum superposition of elementary propositional cases, the truth value of a proposition being in this case a probabilistic value ranging from completely false (0), to completely true (1). 


\section{Eigenlogic: quantum observable logic}

\subsection{Propositional logic in the quantum eigenspace}

A logical proposition is associated to a projection operator in Hilbert space. This operator, being Hermitian, is a quantum observable (here a quantum logical observable). This view is named Eigenlogic (Toffano 2015), (Dubois \& Toffano 2017) and can be summarized as follows:

- eigenvectors in Hilbert space $\Leftrightarrow$ interpretations (input propositional cases)

- logical observables $\Leftrightarrow$ logical connectives

- eigenvalues $\Leftrightarrow$ truth values

The important and original point is that the eigenvalues of the logical observable are the truth-values of the corresponding logical connective.

$$
\boldsymbol{F}=f(0) \boldsymbol{\Pi}_{0}+f(1) \boldsymbol{\Pi}_{1}=\operatorname{diag}[f(0), f(1)]
$$

Where the terms in the development are the 2-dimensional rank-1 projectors $\boldsymbol{\Pi}_{0}$ and $\boldsymbol{\Pi}_{1}$, the cofactors $f(0)$ and $f(1)$ are the eigenvalues (truth values) taking the values $\{0,1\}$. One can define a seed projection operator $\boldsymbol{\Pi}$ that generates all logical observables:

$$
\boldsymbol{\Pi}=\operatorname{diag}(0,1)=\boldsymbol{\Pi}_{1} \quad, \quad \boldsymbol{\Pi}_{0}=\operatorname{diag}(1,0)=\mathbf{I}_{2}-\boldsymbol{\Pi}
$$

This gives the complete set of one-argument logical observables:

$$
\begin{gathered}
\boldsymbol{F}_{A}=0 \boldsymbol{\Pi}_{0}+1 \boldsymbol{\Pi}_{1}=\boldsymbol{\Pi}, \quad \boldsymbol{F}_{\bar{A}}=1 \boldsymbol{\Pi}_{0}+0 \boldsymbol{\Pi}_{1} \\
\boldsymbol{F}_{\perp}=0 \boldsymbol{\Pi}_{0}+0 \boldsymbol{\Pi}_{1}=\mathbb{O}_{2} \quad, \quad \boldsymbol{F}_{T}=1 \boldsymbol{\Pi}_{0}+1 \boldsymbol{\Pi}_{1}=\mathbf{I}_{\mathrm{d}}
\end{gathered}
$$

these operators correspond to: logical projector $A$, its negation $\bar{A}$, contradiction $\perp$ and tautology $T$. Negation corresponds to complementation the respective operator is:

$$
\overline{\boldsymbol{F}}=\mathbf{I}_{d}-\boldsymbol{F}
$$

where $\mathbf{I}_{d}$ is the identity operator and $d=2^{n}$ is the dimension of the considered vector space. For the one-argument case, $n=1$, the eigenvectors $|0\rangle$ and $|1\rangle$ are the 2-dimensional orthonormal vectors:

$$
|0\rangle=(1,0)^{t}, \quad|1\rangle=(0,1)^{t}
$$

Vectors $|0\rangle$ and $|1\rangle$, form the canonical basis, and are often represented in quantum mechanics as qubits corresponding to points on the Bloch sphere (see Figure 1). The choice of the position of the value 1 for the eigenvectors $|0\rangle$ and $|1\rangle$, follows quantum information conventions for a qubit in the computational basis (Nielsen and Chuang 2000).

For two arguments the eigenvectors also form a complete canonical basis. The eigenvectors are named $|a, b\rangle$ where the arguments $a$ and $b$ take the values $\{0,1\}$ and represent one of the four possible cases (logical interpretations) for the input of the logical connective, they are calculated using the Kronecker product of the one-qubit state vectors $|0\rangle$ and $|1\rangle$, explicitly: 


$$
\begin{array}{ll}
|00\rangle=|0\rangle \otimes|0\rangle=(1,0,0,0)^{t}, \quad|01\rangle=|0\rangle \otimes|1\rangle=(0,1,0,0)^{t} \\
|10\rangle=|1\rangle \otimes|0\rangle=(0,0,1,0)^{t}, \quad|11\rangle=|1\rangle \otimes|1\rangle=(0,0,0,1)^{t}
\end{array}
$$

The two-argument logical observables can then be developed in a similar way:

$$
\boldsymbol{F}_{2}=\operatorname{diag}[f(0,0), f(0,1), f(1,0), f(1,1)]
$$

There are in total $2^{2^{n}}$ logical connectives for a $n$-argument (arity) system. For $n=1$ this leads to the four connectives described above. For $n=2$ one has 16 binary logical connectives: conjunction ( $\wedge$ , $A N D)$; inclusive disjunction $(\mathrm{V}, O R)$; exclusive disjunction $(\oplus, X O R)$; logical projectors $(A, B)$; material implication $\Rightarrow$; Sheffer stroke $(\uparrow, N A N D)$; tautology $(T) \ldots$ The remaining ones can be systematically derived by the classical theorems of logic, all are listed on Table 1.

All logical connectives are uniquely characterized by truth tables i.e. by their logical semantics. The logical projectors of the 2-ragument logical system are defined by the following operators:

$$
\boldsymbol{A}=\boldsymbol{\Pi} \otimes \mathbf{I}_{2}=\operatorname{diag}(0,0,1,1) \quad, \quad \boldsymbol{B}=\mathbf{I}_{2} \otimes \boldsymbol{\Pi}=\operatorname{diag}(0,1,0,1)
$$

\begin{tabular}{|c|c|c|c|}
\hline $\begin{array}{c}\text { connective for Boolean } \\
\text { A, B }\end{array}$ & $\begin{array}{l}\text { truth table }\{F, T\} \text { : } \\
\{0,1\} \text { or }\{+1,-1\}\end{array}$ & $\begin{array}{c}\{0,1\} \text { projective } \\
\text { logical observable }\end{array}$ & $\begin{array}{r}\{+1,-1\} \text { isometric } \\
\text { logical observable }\end{array}$ \\
\hline False F, Contradict. $\perp$ & F F F F & 0 & $+I$ \\
\hline NOR & T F F F & $I-A-B+A B$ & $(1 / 2)(+\boldsymbol{I}-\boldsymbol{U}-\boldsymbol{V}-\boldsymbol{U} \boldsymbol{V})$ \\
\hline$A \nLeftarrow B$ & F T F F & $B-A B$ & $(1 / 2)(+\boldsymbol{I}-\boldsymbol{U}+\boldsymbol{V}+\boldsymbol{U} \boldsymbol{V})$ \\
\hline$\neg \mathrm{A}$ & T T F F & $I-A$ & $-\boldsymbol{U}$ \\
\hline$A \nRightarrow B$ & F F T F & $A-A B$ & $(1 / 2)(+I+U-V+U V)$ \\
\hline$\neg \mathrm{B}$ & T F T F & $I-B$ & $-V$ \\
\hline $\mathrm{XOR} ; \mathrm{A} \oplus \mathrm{B}$ & F T T F & $A+B-2 A B$ & $U V=Z \otimes Z$ \\
\hline NAND ; ATB & $\mathrm{TTTF}$ & $\mathrm{I}-A B$ & $(1 / 2)(-\boldsymbol{I}-\boldsymbol{U}-\boldsymbol{V}+\boldsymbol{U} \boldsymbol{V})$ \\
\hline AND ; $A \wedge B$ & F F F T & $A B=\Pi \otimes \Pi$ & $(1 / 2)(+I+\boldsymbol{U}+\boldsymbol{V}-\boldsymbol{U} \boldsymbol{V})$ \\
\hline$A \equiv B$ & T F F T & $I-A-B+2 A B$ & $-U V$ \\
\hline $\mathrm{B}$ & F T F T & $B=I \otimes \Pi$ & $V=I \otimes Z$ \\
\hline$A \Rightarrow B$ & $\mathrm{~T} T \mathrm{~F} \mathrm{~T}$ & $I-A+A B$ & $(1 / 2)(-I-U+V-U V)$ \\
\hline$A$ & F F T T & $A=\Pi \otimes I$ & $\boldsymbol{U}=\mathbf{Z} \otimes \mathbf{I}$ \\
\hline$A \Leftarrow B$ & T F T T & $I-B+A B$ & $(1 / 2)(-I+U-V-U V)$ \\
\hline OR ; AvB & F T T T & $A+B-A B$ & $(1 / 2)(-I+U+V+U V)$ \\
\hline True T, Tautology & T T T T & I & -1 \\
\hline
\end{tabular}

The complete family of the projection operators corresponding to the 16 two-argument connectives are shown on Table 1.

Table 1. The sixteen two-argument binary logical observables and their truth-tables. 
Using the following linear bijection between the projection observable $\boldsymbol{F}$ and a reversible observable $\boldsymbol{G}$ :

$$
\boldsymbol{G}=\boldsymbol{I}_{d}-2 \boldsymbol{F}
$$

one obtains a logical binary system using now the alphabet $\{+1,-1\}$. Observables $\boldsymbol{F}$ and $\boldsymbol{G}$ commute, so they have the same system of eigenvectors. The eigenvalues that correspond to the truth values, respectively "false" and "true", are now respectively +1 and -1 .

The generating observable for this system is the Pauli matrix $\sigma_{z}=Z$ :

$$
\boldsymbol{Z}=\boldsymbol{I}_{2}-2 \boldsymbol{\Pi}_{1}=\left(\begin{array}{cc}
+1 & 0 \\
0 & -1
\end{array}\right)
$$

For two arguments, in the $\{+1,-1\}$ system , the logical dictators $\boldsymbol{U}$ and $\boldsymbol{V}$ (the equivalent of the logical projectors $\boldsymbol{A}$ and $\boldsymbol{B}$ for the system $\{0,1\})$ are:

$$
\boldsymbol{U}=\boldsymbol{Z} \otimes \mathbf{I}_{2}=\operatorname{diag}(+1,+1,-1,-1) \quad, \quad \boldsymbol{V}=\mathbf{I}_{2} \otimes \mathbf{Z}=\operatorname{diag}(+1,-1,+1,-1)
$$

Negation in the $\{+1,-1\}$ system is simply obtained by multiplying by -1 :

$$
\overline{\boldsymbol{G}}=-\boldsymbol{G}
$$

The complete family of the self-inverse logical observables $\boldsymbol{G}$ corresponding to the 16 two-argument connectives are shown on Table 1.

\subsection{Fuzzy logic using non-eigenvectors.}

What happens when the quantum state is not one of the eigenvectors of the logical system? In quantum mechanics one can always express a quantum state-vector $|\psi\rangle$ as a combination on a complete orthonormal basis. One can, for example, choose the canonical eigenbasis of the logical observable family, leading to the development:

$$
|\psi\rangle=c_{00}|00\rangle+c_{01}|01\rangle+c_{10}|10\rangle+c_{11}|11\rangle
$$

When only one of the coefficients is not zero, then one obtains a fixed interpretation for the propositional system corresponding to one of the eigenstates which is the case of the preceding discussion.

Fuzzy logic (Zadeh 1965) deals with truth values that can take values between 0 and 1 , so the truth of a proposition can lie between "completely true" and "completely false". When more than one coefficient in the development of $|\psi\rangle$ is non-zero one can consider a fuzzy interpretation. In this case the quantum state $|\psi\rangle$ can be understood as a superposition of interpretations. Intuitively speaking the superposition principle of vector states considered as logical entities is analogous to a fuzzy logic formulation using fuzzy sets where an entity can belong to more than one set.

For a projective observable $\boldsymbol{F}$ measured in the context of the quantum state $|\psi\rangle$ the mean value (Born rule) leads directly to a probability measure:

$$
p_{|\psi\rangle}=\langle\psi|\boldsymbol{F}| \psi\rangle=\operatorname{Tr}(\boldsymbol{\rho} \cdot \boldsymbol{F}) \quad \text { with } \quad \boldsymbol{\rho}=|\psi\rangle\langle\psi| \quad \text { the density matrix }
$$


The obtained mean value of the logical observable $\boldsymbol{F}$ is thus a fuzzy measure of the truth of a logical proposition in the form of a fuzzy membership function $\mu$.

For one-argument an arbitrary 2-dimensional quantum state can be written as:

$$
|\phi\rangle=\sin \alpha|0\rangle+e^{i \beta} \cos \alpha|1\rangle
$$

where the angles $\alpha$ and $\beta$ are real numbers.

The corresponding membership function corresponds to the quantum mean value of the logical projector observable $\boldsymbol{A}=\boldsymbol{\Pi}$ :

$$
\mu(a)=\langle\phi|\Pi| \phi\rangle=\cos ^{2} \alpha
$$

Using the angular transformation $\alpha=\theta / 2$ and $\beta=\varphi / 2$ one can represent the angles $\theta$ and $\varphi$ on the Bloch sphere (see Figure 1).

Figure 1: Bloch sphere with the general qubit quantum state $|\phi\rangle$ characterized by angles $\theta$ and $\varphi$.

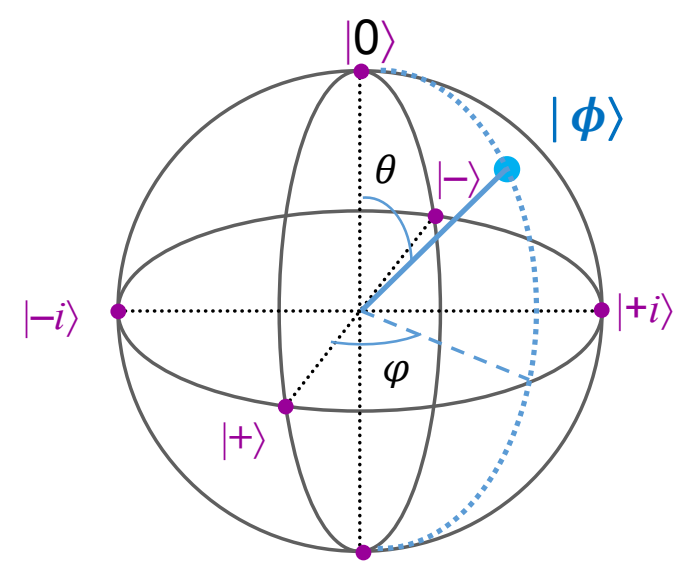

$|1\rangle$

\subsection{Multi-valued Eigenlogic.}

The total number of logical connectives for a system of $m$ values and $n$ arguments is the combinatorial number $m^{m^{n}}$ (Post 1921). In the case of a 3-valued 2-argument system this number becomes $3^{3^{2}}=$ 19683. This large number illustrates the fact that using the totality of the possible logical connectives in a single mathematical framework is intractable beyond the binary case, but some special connectives play an important role and will be used hereafter.

The recently observed revival of interest in applying multi-valued logic (MVL) to the description of quantum phenomena is also closely related to fuzzy logic. MVL is of interest to engineers involved in various aspects of information technology and has a long history, for example it is at the basis of the programming language HDL (Hardware Description Language). 
MVL can be naturally associated to the physical quantity: angular momentum (Dubois \& Toffano 2017). The observable of the $z$ component of the $l=1$ quantum orbital angular momentum observable is characterized by the following matrix possessing three distinct eigenvalues:

$$
\boldsymbol{L}_{z}=\hbar \boldsymbol{\Lambda}=\hbar \operatorname{diag}(+1,0,-1)
$$

One can associate these eigenvalues to the three logical truth values:

$$
\text { "false" } F \equiv+1, \quad \text { "neutral/indeterminate" } N \equiv 0, \quad \text { "true" } T \equiv-1
$$

In this case the logical observables can be expressed as a unique development by spectral decompositions over the three rank-1 projection operators:

$$
\Pi_{+1}=\frac{1}{2} \Lambda(\Lambda+1) \quad, \quad \Pi_{0}=I-\Lambda^{2} \quad, \quad \Pi_{-1}=\frac{1}{2} \Lambda(\Lambda-1)
$$

\section{Eigenlogic applied to Quantum Robot Braitenberg Vehicles}

\subsection{Braitenberg Vehicles (BV)}

Valentino Braitenberg was a Cyberneticist and former director at the Max Planck Institute for Biological Cybernetics in Tübingen. In his book Vehicles (Braitenberg 1986) he describes various thought experiments which involve simple machines (vehicles) that consist of sensors, motors and wheels.

Braitenberg prompts the readers to imagine the behaviours of these vehicles under different internal connections between the sensors and the motors of the vehicles. He then links these behaviours to specific emotions that can be attributed to such behaviours. This approach is referred to by the author as law of uphill analysis and downhill invention. According to this principle it is far easier to create machines that exhibit complex behaviour based on simple structures than it is to try to design their structures based on behavioural observations and interpretations. The very simple mobile vehicles use basic sensory-motor connections to produce seemingly cognitive behaviours that display complex lifelike behaviours far beyond those which would be expected considering their simple logical structure.

A Braitenberg vehicle (BV) is an agent that can autonomously move around. The sensors are connected by circuits to actuators controlling the motion of two wheels on the rear of the vehicle (see Fig. 2). Depending on the sensor-motor wiring, they appear to accomplish certain situations and to avoid others, changing course when the situation changes. Several elementary vehicles can be considered:

- BV-2a (fear): turns away from the light if one sensor is activated more than the other.

- $\quad B V-2 b$ (aggress): if the light source is near either sensor, the vehicle will go toward it.

- BV-3a (love): continues to go until it finds a light source, then slows to a stop.

- BV-3b (explore): goes to the nearby light source, keeps an eye open to drive to a stronger source.

The practical realization of BV's is generally based on simple Boolean logic circuits. It is therefore interesting to extend the design to multi-valued and fuzzy logic. 


\subsection{Braitenberg Vehicles and quantum-like behaviour}

Most of the interest in quantum computing is due to quantum algorithms, specifically the Grover query and Shor's factoring algorithms (Nielsen \& Chuang 2000), resulting in an advantage for computational speed-up over classical computing. Paul Benioff who was the first to propose the idea of a quantum Turing machine in 1980 introduced also another paradigm: the theoretical principle of a quantum robot (Benioff 1998) as a first step towards a quantum mechanical description of systems that are aware of their environment and make decisions. In this view two configurations are considered:

I. A stand-alone quantum circuit aboard the robot, the quantum computer does not interact with the environment (stimuli, other robots...)

II. A collection of interacting quantum robots in their environment, the global system can then be considered as a single quantum machine.

Currently, much research is undertaken on the analysis of the interactions between quantum computers and their environment. These interactions are generally considered as a source of noise or errors that must be minimized or corrected by using, for example, quantum error correction techniques (Nielsen and Chuang 2000). On the other hand in the present approach the interaction between a quantum robot and its environment has to be considered as an essential part of the overall dynamics of the system.

The research team led by Marek Perkowski has designed robots based on BV's, using quantum gates and also introducing control, fuzziness and higher than binary valued logic (Raghuvanshi and Perkowski 2010). Inspired by these researches the applications presented here seek quantum-like behaviours using the original formulation of Eigenlogic. The scope of the investigations presented hereafter is not to build actual quantum devices but to investigate the design possibilities using the quantum formalism. This is what is intended by the term quantum-like which is less stringent than the more widespread term quantumness. It has to be outlined that the study of quantum-like phenomena outside physics has been one of the principal motivations of the Quantum Interaction community over the last years (Haven \& Khrennikov 2013).

\subsection{Quantum robots: binary BV}

The binary alphabet $\{+1,-1\}$ is well adapted to make analogies with the vehicle's behavior, it has a natural correspondence with inhibition (negative, - ) and excitation (positive, + ). The BV's sensors $S L$ and $S R$ (see Figure 2) represent the inputs and the actuators $M L$ and $M R$ correspond to the 2-argument operators (logical dictators):

$$
\boldsymbol{U}=\operatorname{diag}(1,1,-1,-1) \quad, \quad \boldsymbol{V}=\operatorname{diag}(1,-1,1,-1)
$$

The different possible logical combinations (Braitenberg 1986) are given on Table 2. 
Table 2. logical observables for BV actuators.

\begin{tabular}{|l|c|c|}
\hline Braitenberg Vehicle\Actuator & ML & MR \\
\hline BV2a (fear) & $-\boldsymbol{U}$ & $-\boldsymbol{U}$ \\
\hline BV2b (aggress) & $-\boldsymbol{U}$ & $-\boldsymbol{U}$ \\
\hline BV3a (love) & $+\boldsymbol{U}$ & $+\boldsymbol{V}$ \\
\hline BV3b (explore) & $+\boldsymbol{V}$ & $+\boldsymbol{U}$ \\
\hline
\end{tabular}

It is possible to use other logical observables (see Table 1) leading to different logical combinations corresponding to more complex behaviours.

Figure 2: A binary quantum Braitenberg Vehicle, with left and right sensors (SL, SR), left and right motors (ML, MR) and left and right wheels (WL, WR).

WL

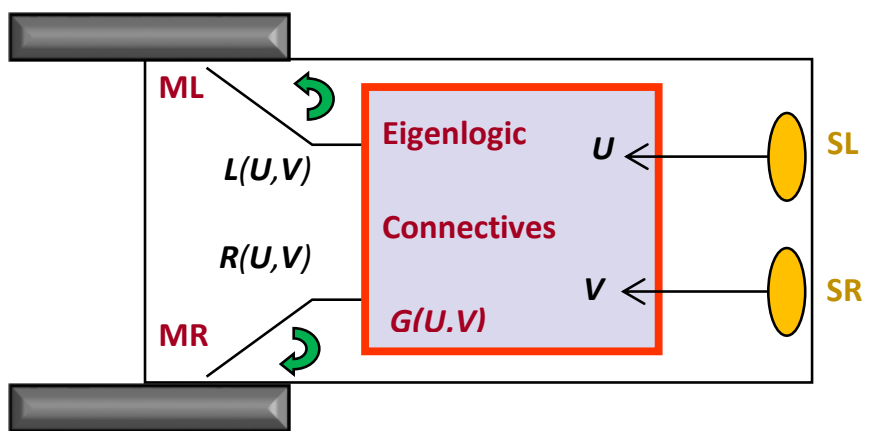

\subsection{Quantum robots: multivalued BV.}

In another configuration the sensors $S L$ and $S R$ can be characterized by multivalued ternary input logical observables $\boldsymbol{U}$ and $\boldsymbol{V}$. For this purpose it can be interesting to use an alphabet with three positive values $\{0,1,2\}$ in the following interpretation:

$$
\text { "no light" } \equiv 0 \text {, "weak light" } \equiv 1, \quad \text { "strong light" } \equiv 2
$$

Complex behaviours can then be achieved using the Min and Max decision connectives. From the formulation given above based on interpolation methods (Dubois and Toffano 2017) one can derive the corresponding logical observables for the alphabet $\{0,1,2\}$ : 


$$
\begin{gathered}
\boldsymbol{M i n}_{3\{0,1,2\}}=\boldsymbol{U}+\boldsymbol{V}+\boldsymbol{U}^{2} \boldsymbol{V}+\boldsymbol{V}^{2} \boldsymbol{U}-\frac{1}{2} \boldsymbol{U}^{2} \boldsymbol{V}^{2}-\frac{5}{2} \boldsymbol{U} \boldsymbol{V}=\operatorname{diag}(0,0,0,0,1,1,0,1,2) \\
\boldsymbol{M a x}_{3\{0,1,2\}}=\frac{5}{2} \boldsymbol{U} \boldsymbol{V}+\frac{1}{2} \boldsymbol{U}^{2} \boldsymbol{V}^{2}-\boldsymbol{U}^{2} \boldsymbol{V}-\boldsymbol{V}^{2} \boldsymbol{U}=\operatorname{diag}(0,1,2,1,1,2,2,2,2)
\end{gathered}
$$

The Boolean binary logical system using values $\{0,1\}$ is included in the ternary logical system $\{0,1,2\}$ indicated above and it easy to verify that reducing to binary logic, the Max connective becomes the inclusive disjunction $O R$ and Min the conjunction AND.

The simple example illustrated above is mainly of pedagogical value. It has to be emphasized that the non-classical implications of three-valued Eigenlogic would be a project in itself but is beyond the scope of the present paper. It could bring up new issues and complex behaviour patterns that go beyond the possibility of classical non-quantum propositional logic.

For example three-valued logic has considerable scope and potential for dealing with issues such as measurement and autonomy. Hans Reichenbach (Reicenbach 1948) proposes a three-valued logic to deal with "the principle of causal anomaly in quantum mechanics" which is intimately connected with the measurement problem of non-commuting observables. For this reason the above interpretation includes a widening of the definition of meaning: a statement is meaningful if it is verifiable as true, false and indeterminate.

Furthermore to deal with the intrinsic paradox in self-reference, Francisco Varela (Varela 1975) introduced a third state, "the autonomous state" alongside the marked and unmarked states of Spencer-Brown's laws of Form (Spencer \& Brown 1969). In this language, the new state refers to reentry, and leads on to cybernetic concepts such as operational closure, self-reflection, self-organization and autonomy. A challenging question could be: can multivalued Eigenlogic actually provide selfreferential robots?

Figure 3: A multivalued quantum Braitenberg Vehicle with multivalued Min and Max quantum decision circuits.

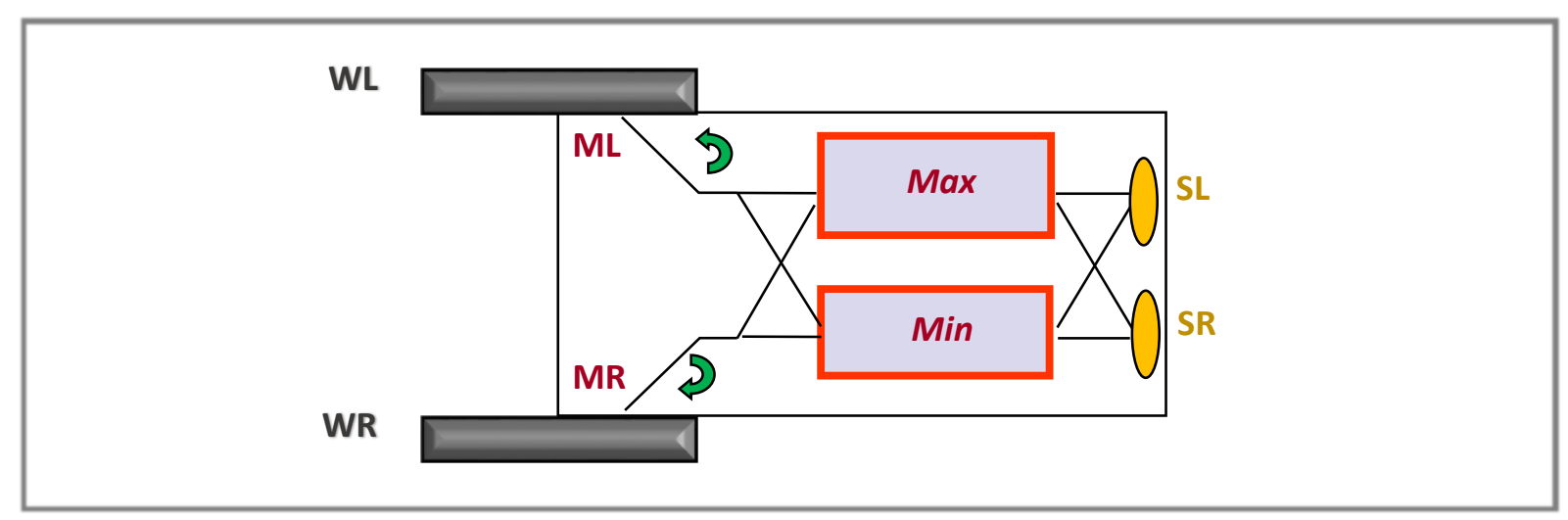

\subsection{Quantum robots: fuzzy-logic BV.}

Processes which consider an interaction with the environment using a quantum robot approach have been considered in (Raghuvanshi \& Perkowski 2010). In (Rigatos \& Tzafestas 2006), a method for implementing quantum associative memories using fuzzy logic was proposed where the probability of each state is represented by a fuzzy membership function. 
There are similarities between neural network models and quantum computing formalism. In particular, it is possible to implement quantum learning algorithms dedicated to fuzzy qubits (Hannachi et al 2007). In this case the weighted sums of inputs of a neuron correspond to the superposition of quantum states at the input of a quantum circuit and the quantum wave function collapse corresponds to the threshold activation of a neuron.

The Eigenlogic observables defined before can be put in a fuzzy logic configuration, i.e. when inputs from the sensors are not eigenstates. One can for example calculate the fuzzy membership functions for the complement $\mu(\bar{a})$ or for the Min and Max connectives: $\mu($ Min $)$ and $\mu(\operatorname{Max})$. These functions can be implemented in the processor preceding the actuators $M L$ and $M R$ (see Figure 2 and Figure 3 ).

Another strategy is to characterize "fuzzy motions" represented by a set of input states (qubits) corresponding to the possible directions of the robot.

$$
\begin{gathered}
|b\rangle=|0\rangle=(1,0)^{t}, \quad|f\rangle=|1\rangle=(0,1)^{t} \\
|l\rangle=|+\rangle=\frac{1}{\sqrt{2}}(|0\rangle+|1\rangle)=\frac{1}{\sqrt{2}}(1,1)^{t}, \quad|r\rangle=|-\rangle=\frac{1}{\sqrt{2}}(|0\rangle-|1\rangle)=\frac{1}{\sqrt{2}}(1,-1)^{t}
\end{gathered}
$$

These states are represented on the Bloch sphere in Figure 4.

One notices that $|b\rangle$ and $|f\rangle$ are eigenstates of the Eigenlogic system but $|l\rangle$ and $|r\rangle$ are not.

A general qubit state vector with real coefficients is:

$$
|s\rangle=s_{0}|0\rangle+s_{1}|1\rangle
$$

this state corresponds to the angle $\theta$ on the Bloch sphere with the respective coefficients

$$
s_{0}=\cos \frac{\theta}{2} \quad, \quad s_{1}=\sin \frac{\theta}{2} .
$$

The associated fuzzy membership functions $\mu$ can then be derived for different motion configurations. The one corresponding to forward motion $\mu_{f}$ in the state $|s\rangle$ is simply:

$$
\mu_{f}(s)=\langle s|\Pi| s\rangle=s_{1}^{2}
$$

Leading for the left and right turns to:

$$
\mu_{f}(l)=\mu_{f}(r)=\frac{1}{2}
$$

The coefficients $s_{0}$ and $s_{1}$ can thus be extracted from the Eigenlogic fuzzy membership function $\mu$, allowing to estimate the most suitable input qubit states for the desired motion. This process could be designed dynamically for control and steering of the robot following the method proposed in (Hannachi et al. 2007) and (Rybalov et al. 2010). 


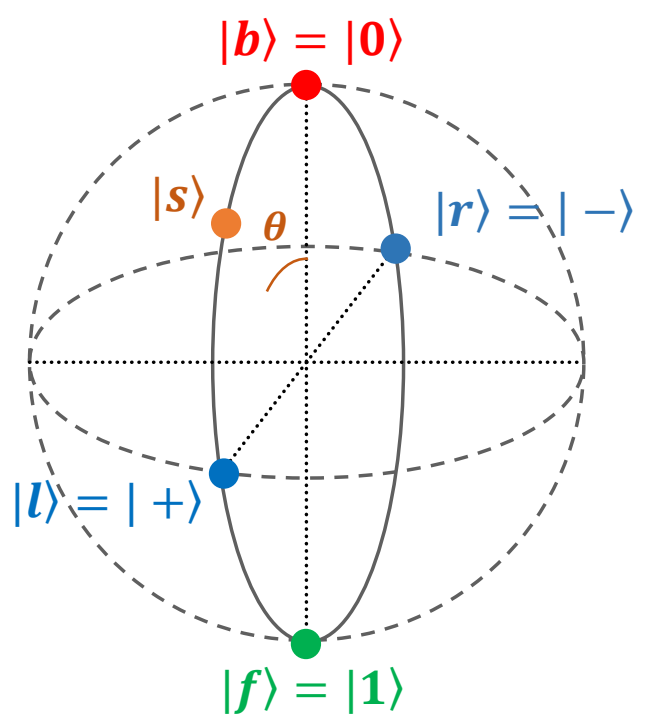

This same method can then be applied to a compound state $|p, q\rangle$ corresponding to a 2-qubit state. The following cases can be considered:

$$
\begin{gathered}
\text { "step forward" /"turn left": }|f, l\rangle=\frac{1}{\sqrt{2}}(|10\rangle+|11\rangle) \\
\text { "turn left"/"turn right": }|l, r\rangle=\frac{1}{2}(|00\rangle-|01\rangle+|10\rangle-|11\rangle)
\end{gathered}
$$

The fuzzy membership function corresponding to the logical connective for material implication $(\Rightarrow)$ is then used for the decision of the vehicle motion options such as "step backwards", "step forwards", "turn left" and "turn right". Here the corresponding Eigenlogic observable $\boldsymbol{F}_{\Rightarrow}$, given in Table 1, and corresponding to logical implication will be used:

$$
\boldsymbol{F}_{\Rightarrow}=\operatorname{diag}(1,1,0,1)=(\mathbf{I}-\boldsymbol{\Pi} \otimes \mathbf{I}+\boldsymbol{\Pi} \otimes \boldsymbol{\Pi})
$$

For "forward/left" $\rightarrow$ "forward" the membership function value becomes:

$$
\mu_{f}((f, l) \rightarrow f)=\langle f, l|(\mathbf{I}-\boldsymbol{\Pi} \otimes \mathbf{I}+\boldsymbol{\Pi} \otimes \boldsymbol{\Pi})| f, l\rangle=\left(\frac{1}{2}+\frac{1}{2}\right)-\left(\frac{1}{2}+\frac{1}{2}\right)+\frac{1}{2}=\frac{1}{2}
$$

and for "left/right" $\rightarrow$ "forward" the membership function value becomes:

$$
\mu_{f}((l, r) \rightarrow f)=\langle l, r|(\mathbf{I}-\boldsymbol{\Pi} \otimes \mathbf{I}+\boldsymbol{\Pi} \otimes \boldsymbol{\Pi})| l, r\rangle=1-\left(\frac{1}{4}+\frac{1}{4}\right)+\frac{1}{4}=\frac{3}{4}
$$

There are different classical formulations of fuzzy implication, mostly issued from multivalued logic. The Kleene-Dienes and Łukasiewicz implications, $\mu_{f}{ }^{K D}$ and $\mu_{f}{ }^{L}$ give resp:

$$
\begin{gathered}
\mu_{f}^{K D}((f, l) \rightarrow f)=\max \left(1-\mu_{f}(f), \mu_{f}(l)\right)=\max \left(1-1, \frac{1}{2}\right)=\frac{1}{2} \\
\mu_{f}{ }^{K D}((l, r) \rightarrow f)=\max \left(1-\frac{1}{2}, \frac{1}{2}\right)=\frac{1}{2} \\
\mu_{f}^{L}((f, l) \rightarrow f)=\min \left(1-\mu_{f}(f)+\mu_{f}(l), 1\right)=\min \left(1-1+\frac{1}{2}, 1\right)=\frac{1}{2} \\
\mu_{f}{ }^{L}((l, r) \rightarrow f)=\min \left(1-\frac{1}{2}+\frac{1}{2}, 1\right)=1
\end{gathered}
$$


When comparing these with the preceding Eigenlogic results, the first case $(f, l)$ shows the same result whereas the second: $(l, r)$ is different for all three forms.

\subsection{Quantum robots: contextual BV using superposition and entanglement.}

Another idea presented in (Raghuvanshi \& Perkowski 2010) consists in mapping emotions directly onto the Bloch sphere. The proposed concept of quantum sphere of emotions (Figure 5) inspired by the "Wheel of Emotions" (Plutchik 2001) allows for the representation of multiple emotions represented by phases in Hilbert space. The idea is that an emotion is formed by the combination of 3 components: active/passive behaviour, corresponding the $x$ axis of the Bloch sphere, positive/negative emotions, related to the $y$ axis and intensity, corresponding to the $z$-axis.

Due to the quantum-like nature (state vectors) of these inputs, emotions can be superposed leading to a complex description of the robot emotional state. Therefore one can define a methodology for associating qubit state vectors characterized by their angles with emotions. The internal emotional state of the robot can then be designed using standard quantum logic gates. For instance, single-qubit operators (Hadamard, Pauli and other rotations) and two-qubit gates (Control NOT, Control Z...) can be used (Nielsen \& Chuang 2000).

It has to be outlined that this view leads to contextuality because once given the emotional representation on the sphere, even if the logical operators are characterized by their specific logical connective. The result for the fuzzy membership will depend on the choice of the state vector basis for the robot. In Eigenlogic this corresponds to a contextual logical interpretation.

Figure 5: Quantum sphere of emotions (Raghuvanshi and Perkowski 2010)

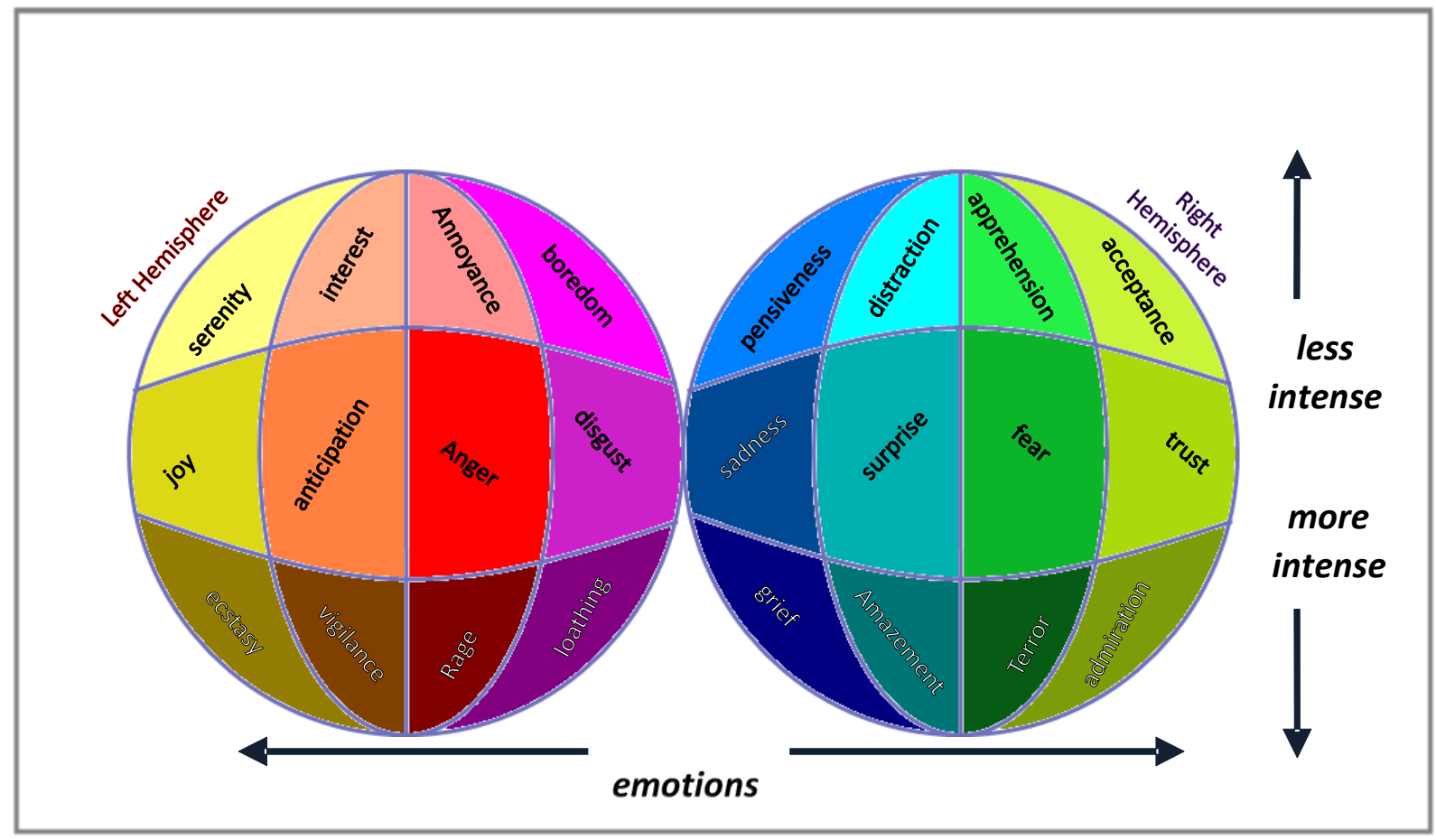


Non-separable or entangled input sates can also be considered in this model. The representation on a single Bloch sphere is no more possible, one has to consider higher dimensional geometry. One of the simplest forms of a completely entangled state resulting from the composition of two qubits is the singlet state represented by the state vector:

$$
|\operatorname{sing}\rangle=\frac{1}{\sqrt{2}}(|01\rangle-|10\rangle)
$$

It is easily verified that this state cannot be put into a separable Kronecker product $\otimes$ as was the case for the compound states discussed above. But one can still use the preceding methods for calculating fuzzy membership functions. The fuzzy measure for implication on the singlet state is given by:

$$
\mu_{f}((\operatorname{sing}) \rightarrow f)=\left\langle\operatorname{sing}\left|\boldsymbol{F}_{\Rightarrow}\right| \operatorname{sing}\right\rangle=\langle\operatorname{sing}|(\mathbf{I}-\boldsymbol{\Pi} \otimes \mathbf{I}+\boldsymbol{\Pi} \otimes \boldsymbol{\Pi})| \operatorname{sing}\rangle=1-\frac{1}{2}+0=\frac{1}{2}
$$

The interpretation of this result is challenging because no set of purely local variables can be used to describe the resulting state and its character is essentially nonlocal.

It has to be outlined that the issue of nonlocality and entanglement is nowadays considered more ubiquitous (Vedral 2014) than initially assumed and is also natural in the framework of Eigenlogic. For example 8 among 16 of the 2-qubit Eigenlogic self-inverse operators $\boldsymbol{G}$ are nonlocal, i.e. they are not a Kronecker product of two one-qubit operators.

In Table 1 these correspond to the self-inverse operators possessing 3 degenerate and one single eigenvalue, each eigenvalue corresponding to the truth values. These operators are the conjunction $\boldsymbol{G}_{\mathrm{AND}}=\operatorname{diag}(1,1,1,-1)$, its logical complement $\boldsymbol{G}_{\mathrm{NAND}}=\operatorname{diag}(-1,-1,-1,1)$, disjunction $\boldsymbol{G}_{\mathrm{OR}}=$ $\operatorname{diag}(1,-1,-1,-1)$, its complement $\boldsymbol{G}_{\mathrm{NOR}}=\operatorname{diag}(-1,1,1,1)$, also the material implication $\boldsymbol{G}_{\Rightarrow}=$ $\operatorname{diag}(-1,-1,1,-1)$, its converse $\boldsymbol{G}_{\models}=\operatorname{diag}(-1,1,-1,-1)$ and the complements $\boldsymbol{G}_{\nRightarrow}=$ $\operatorname{diag}(1,1,-1,1)$ and $\boldsymbol{G}_{\sharp}=\operatorname{diag}(1,-1,1,1)$.

From a quantum computing perspective non-local operators are essential for building universal quantum gates. The association of the Control-NOT gate, which is a non-local 2-qubit gate, with onequbit gates constitutes a universal quantum gate set. A method based on Eigenlogic has recently been developed for designing such gates (Toffano \& Dubois 2017). It is also important to notice that only these non-local control gates have an entangling power when applied on qubits that are not eigenstates.

It has been suggested that using these gates in a fuzzy logic configuration makes it possible to implement quantum associative memory learning and retrieval algorithms (Ventura \& Martinez 2000), (Hannachi et al. 2007).

Recently another quantum-like approach in connection with the functioning of neurons has been proposed in (Khrennikov et al. 2018), where modelling of information processing by neurons was done using the theory of open quantum systems. In particular the process of decision-making was associated to the quantum decoherence effect. It would be interesting to compare methods based on the quantum entangling control gates discussed here-above with this research on neural decision making based on quantum probability and information. This direction of investigation could be motivated by the fact that the decoherence mechanism can also be interpreted by means of quantum control gates (Paz \& Zurek 2002). 
Therefore by means of "experimental" quantum-like approaches testing various configurations of nonseparable input quantum states and by analyzing the corresponding behaviors, one would be able to make associations between the structure of these states and the corresponding observed robot behavior. One could also search for more complex behaviors impossible to obtain using only local separable input qubit states.

\section{Outlook and perspectives}

This work shows a new approach to quantum-like modelling applied to behavioral studies. It stems from recent research which has contributed to the Quantum Interaction community, where the quantum formalism is used outside the field of physics. Research efforts in this community have for example presented global quantum-like approaches for social studies (Haven \& Khrennikov 2013). The approach presented in this paper is also related to quantum-like models that have been proposed for studies in semantics (Galofaro et al. 2017) representing the concepts of individuation, metastability and indetermination combined with classical semiotic theories in order to describe the propagation of semantic characters.

The method shown here proposes the application of a quantum formulation of propositional logic, named Eigenlogic, to robotics. To illustrate this it uses Braitenberg Vehicles, and its central underlying working paradigm, "up-hill analysis, down-hill invention" (Braitenberg 1986), that has attracted much interest in robotics research. The case of multivalued and fuzzy logic operations for control and learning in BVs are discussed showing new perspectives for quantum information processing applied to behavioural agents.

The last examples of fuzzy BV robots in section 3-5 reflect contextuality through superposition and entanglement which are the principal quantum aspects exploited in the Quantum Interaction community. These aspects are considered at the level of the robot's interpretation of its combined input stimuli. The resulting robot's behaviour becomes non-classical and can be qualified as quantumlike. By changing and combining different quantum gates, including entangling gates, to act as controlling functions, the observed changes in the vehicle's behaviour lead to peculiar features. The observed behaviour combines the main BV's emotions (fear, aggression, love and explore) according to the interpretation based on the quantum sphere of emotions. Computer simulations that permit visualization of these complex behaviours resulting from the multiple combination of quantum control gates have been presented in (Araripe et al. 2018). These allow the detection of new Braitenberg vehicle behaviour patterns related to identified emotions and linked to quantum-like effects.

The important scientific domain of quantum information has become one of the greatest research efforts worldwide. Computer science has integrated quantum paradigms in order to propose algorithms outperforming their classical counterparts. In this paper the concept of quantum robots (Benioff 1998) corresponding to quantum systems interacting with their environment is considered. The view here is significantly different from the quantum circuit methods where one tries to isolate quantum behaviour from external perturbations. Here the interaction between a quantum robot and its environment is an essential part of the overall dynamics of the system. This approach has parallels with performative systems and their related strategies to manage complexity. 
Quantum modelling of phenomena outside the classic realm of physics and chemistry is an emergent field of research highly relevant to Cybernetics. In this paper, we exemplify this expansion of quantum formalism to this currently topical area by showing that quantum theory and methods can seamlessly reproduce and extend propositional logic. This is intriguing and indicates the potential of quantum approach for future robotic development.

The object of the Interactions Revolution, an important approach in modern Cybernetics, is dynamic and structural couplings and co-evolution rather than the study of the separate constituents of a system. Its worldview is interactions and relationships between systems and their environments. Today organizations and social systems need increased fluidity and agility to respond to environmental, social, and economic pressures. The Interactions Revolution offers opportunity for digital technologies and transdisciplinary conceptual frameworks. We think that the approach presented here based on logic and on the emerging quantum information field (recently named the Second Quantum Revolution), could help to reach some of these goals.

\section{References}

Araripe Furtado Cunha, R., Sharma, N., Toffano, Z., Dubois, F., "Fuzzy Logic Behavior of QuantumControlled Braitenberg Vehicle Agents", Quantum Interaction Conerence, QI 2018, to be puiblished in Lecture Notes in Computer Science.

Benioff, P., 1998, "Quantum Robots and Environments", Physical Review A, Vol. 58, No.2, pp. 893-904.

Birkhoff, G., von Neumann J., 1936, "The Logic of Quantum Mechanics". The Annals of Mathematics, 2nd Ser., Vol. 37, No 4, pp. 823-843.

Boole, G., 1847, The Mathematical Analysis of Logic. Being an Essay To a Calculus of Deductive Reasoning, Forgotten Books.

Braitenberg, V. 1986, Vehicles: Experiments in Synthetic, Psychology. MIT Press; Cambridge USA

Dubois F., Toffano Z., 2017, "Eigenlogic: A Quantum View for Multiple-Valued and Fuzzy Systems". de Barros J., Coecke B., Pothos E. (eds) Quantum Interaction. QI 2016. Lecture Notes in Computer Science, vol 10106. Springer. https://doi.org/10.1007/978-3-319-52289-0_19

Galofaro, F., Toffano, Z., Doan, B.L., 2017, "A quantum-based semiotic model for textual semantics", Kybernetes, Vol. 47 Issue: 2, pp.307-320, https://doi.org/10.1108/K-05-2017-0187

Feynman, R., 1965, The Feynman Lectures on Physics - vol. III, Addison Wesley, p. 3-2.

Halperin, T., 1981, “Boole's Algebra isn't Boolean Algebra. A Description Using Modern Algebra, of What Boole Really Did Create", Mathematics Magazine, Vol. 54, N0.4, pp. 172-184. Reprinted, 2000, in A Boole Anthology ed. James Gasser Library, Vol. 291, Springer Verlag.

Hannachi, M. S., Dong, F., Hirota, K., "Emulating Quantum Interference and Quantum Associative Memory Using Fuzzy Qubits", Proc. IEEE Int. Conf. Computational Cybernetics ICCC 2007, 39-45.

Haven, E. and Khrennikov, A., 2013, Quantum Social Science, Cambridge University Press. 
Khrennikov, A., Basieva, I., Pothos, E. M., Yamato, I., 2018, “Quantum probability in decision making from quantum information representation of neuronal states". Scientific Reports, vol. 8, $\mathrm{n}^{\circ}: 16225$.

Nielsen, M.A., Chuang, I.L., 2000, Quantum Computation and Quantum Information, Cambridge University Press, Cambridge.

Paz J.P., Zurek W.H., 2001, "Environment-Induced Decoherence and the Transition from Quantum to Classical", In: Kaiser R., Westbrook C., David F. (eds) Coherent atomic matter waves. Les Houches Ecole d'Ete de Physique Theorique, vol 72. Springer, Berlin, Heidelberg

Post, E., 1921, "Introduction to a General theory of Elementary Propositions", American Journal of Mathematics, 43: pp. 163-185.

Raghuvanshi, A. and Perkowski, M., 2010, "Fuzzy quantum circuits to model emotional behaviors of humanoid robots", IEEE Congress on Evolutionary Computation, 18-23 July 2010, pp. 1-8.

Reichenbach, H., 1948, "The Principle of Anomaly in Quantum Mechanics", Dialectica, Vol 2. Repr Feigl, H., Brodbeck, M., 1953, Readings in the Philosophy of Science, Appleton-Century-Crofts, pp. 509-520.

Rigatos, G. G. and Tzafestas, S.G., 2006, "Quantum learning for neural associative memories," Fuzzy Sets and Systems, Vol. 157, No. 13, pp 1797-1813,

Rybalov, A., Kagan, E., Manor, Y. \& Ben-Gal, I., 2010, “Fuzzy model of control for quantum-controlled mobile robots". In Proc. of 26th IEEE Convention Israel, pp. 19-23.

Spencer-Brown, G., 1969, Laws of Form, Allen \& Unwin, London.

Toffano, Z., 2015, "Eigenlogic in the spirit of George Boole". ArXiv:1512.06632

Toffano, Z.; Dubois, F., 2018, "Interpolating Binary and Multivalued Logical Quantum Gates", Proceedings , 2, 152. https://doi.org/10.3390/ecea-4-05006

Varela, F., 1975, “A calculus for self-reference”, Int. J. General Systems, vol.2, pp. 5-24.

Vedral, V., 2014, "Quantum Entanglement", Nature Physics, vol 10, 256-258 (2014), doi:10.1038/nphys2904

Venn, J., 1881, Symbolic Logic, London: Macmillan and Company.

Ventura, D. \& Martinez, T., 2000, "Quantum associative memory" Information Sciences, vol. 124, Issues 1-4, pp. 273-296

Von Neumann, J., 1932, Mathematical Foundations of Quantum Mechanics. Investigations in Physics, vol. 2, Princeton University Press, Princeton, (transl. 1955).

Wittgenstein, L., 1921, "Logisch-Philosophische Abhandlung", Ann. der Naturphilosophie, Ed. Wilhelm Ostwald, Wien, 1922, Tractatus Logico-Philosophicus, Routledge \& Kegan Paul, London.

Zadeh, L.A., 1965, "Fuzzy sets", Information and Control, 8 (3), 338-353. 


\section{Appendix A - A refresher of some definitions in classical propositional logic}

Here we remind some definitions of classical propositional logic.

A logical connective (also called a propositional logical operator or sentential operator) is a symbol used to connect two or more sentences (of a formal language or a natural language) in a grammatically valid way, so that the value produced by the a compound sentence depends only on that of the original sentences and on the meaning of the connective.

In classical logic, truth functions represent unambiguous symbols and well-formed formulas can be built by joining other well-formed formulas using truth-functional logical connectives.

The method of truth tables, which is due to Wittgenstein in the Tractatus (Wittgenstein 1921), in addition to giving the logical connective matrices, can also be used as an effective logical calculation system. In fact, by using truth tables, one can establish in a completely mechanical way the correctness or non-correctness of all logical propositional forms written in the sentential language.

Almost at the same time Emil Post established in (Post 1921) the consistency and completeness of propositional calculus, in this way logical truth tables representing the semantics of a proposition are axiomatic at the same level as logical connective canonical forms representing their syntax.

Negation is a unary (one input, arity-1) connective. There are sixteen logical duary (two inputs, arity2) binary connectives associating the input truth-values $\{F, T\}$ of the two elementary propositions $A$ and $B$ with a four-digit binary output truth-table (see Table 1 and Figure A). These tables correspond to all the possible choices of arity-2 logical connectives for classical logic. The number of arity- 2 different connectives is exactly $2^{2^{2}}=16$. For higher arity- $n$ one can define other specific connectives: i.e. for arity-3 the majority function. The number of binary connectives grows as $2^{2^{n}}$.

The commonly used logical connectives are Negation (NOT, $\neg$ ), Conjunction (AND, $\wedge$ ) , Disjunction (OR, V), Material implication or Conditional (if...then, $\Rightarrow$ ), Equivalence or Biconditional (if and only if, $\equiv$ ).

Logical redundancy permits to reduce or transform logical connectives. A classical example of redundancy is the logical equivalence between the conditional formula $A \Rightarrow B$ and the disjunction $\neg A \vee B$. This shows that the conditional operator $\Rightarrow$ is not necessary when the negation operator (NOT, $\neg)$ and the disjunction operator (OR, V) are already in use. Alternative connectives are derived using classical theorems of logic such as De Morgan theorem. For example using the De Morgan rule the disjunction of the negated inputs: $\neg A \vee \neg B$ is equivalent to the negation of their conjunction: $\neg(A \wedge B)$.

Logical completeness has an important consequence in propositional logic. It shows the existence of universal connectives generating all the others. In binary propositional logic, the following logical connectives when combined with negation (NOT) are universal: AND, OR, NOR, NAND, $\Rightarrow$, $\nRightarrow$. But the following are not: the logical projectors $A$ and $B$ and their negations $\neg A$ and $\neg B$, Equivalence $\equiv, X O R$, Contradiction F and Tautology T.

A simple way to illustrate this machinery is by the use of Venn diagrams (Venn 1881) as illustrated on Figure A. The dark areas represent "True" and the white areas "False". The truth table of each logical connective (shown at the bottom of each diagram) corresponds to the number of the respective true or false surface sections. Boolean connectives use the non-negative truth-values $\{0,1\}$. Manipulating positive surface measures in Venn diagrams is thus natural when considering Boolean functions.

Venn diagrams have a direct correspondence with set theory and can thus be directly associated to the projection operators as presented here in Eigenlogic. A probabilistic interpretation is also 
straightforward in terms of Venn diagrams, this is often used in Information theory when discussing conditional (Bayesian) probabilities and entropies.

In multivalued logic (higher than binary) logical connectives are also naturally defined. As was shown by E. Post in (Post 1921), for an $m$-valued arity- 2 system one also has two universal connectives. In the case of Post logic (the logical values are the successive positive integers starting from 0 ) these are the general negation (cyclic permutation of successive values $a_{i} \rightarrow a_{i+1}$ ) and the MAX operator (which takes the highest of two input values). For binary values cyclic permutation reduces to negation (complementation) and the MAX becomes the disjunction (OR, $\mathrm{V})$. The exact number of logical connectives for an $m$-valued arity- $n$ system is $m^{m^{n}}$.

Figure A: Venn diagrams of the four arity-1 and of the 16 arity-2 binary logical connectives.

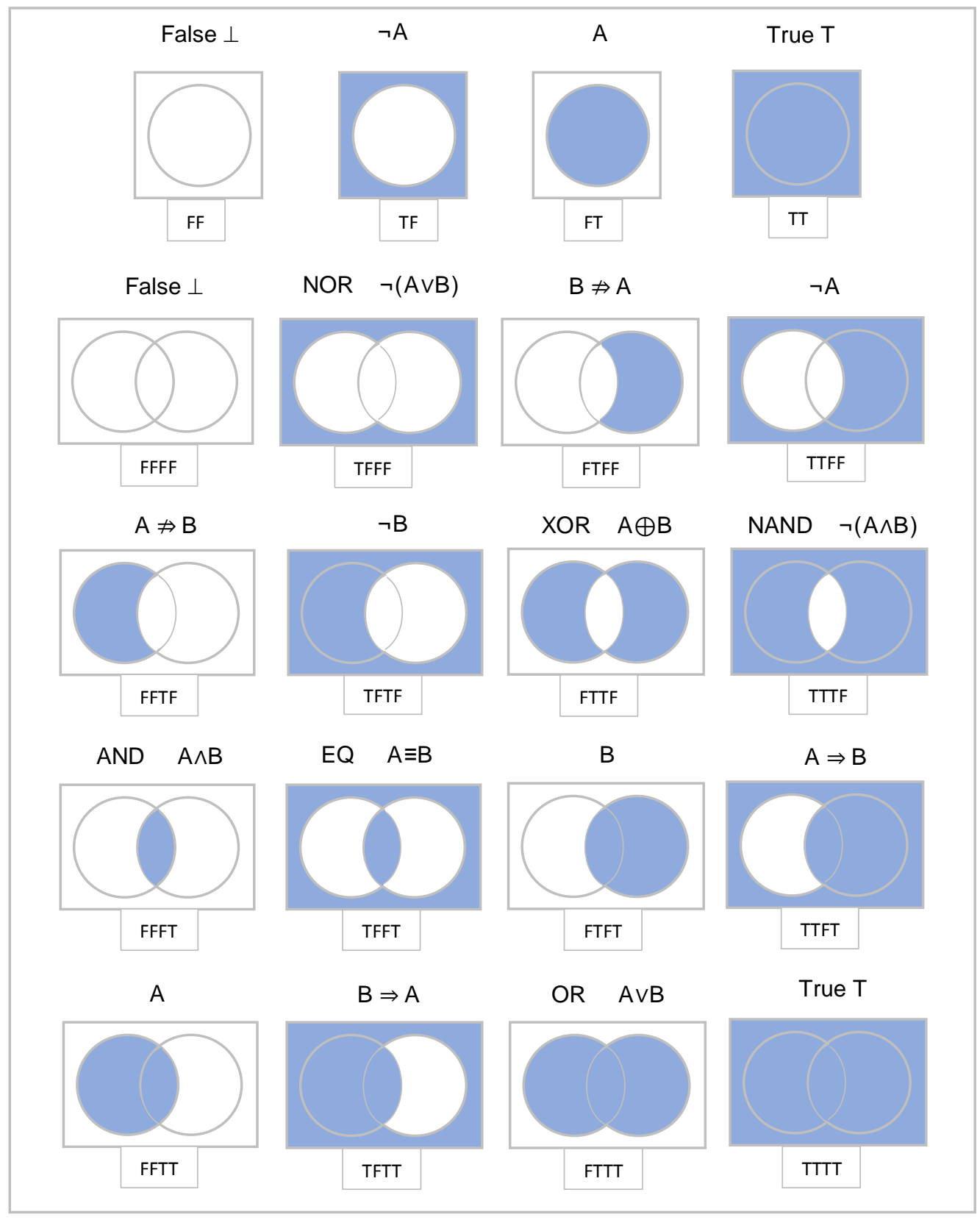

